\title{
Polymer-Micelle Incarcerated Scandium as a Novel Polymer-Supported Catalyst for High-Throughput Organic Synthesis
}

\author{
Masahiro Takeuchi, Ryo Akiyama, and Shū Kobayashi* \\ Graduate School of Pharmaceutical Sciences, The University of Tokyo, The HFRE Division, \\ ERATO, Japan Science and Technology Agency (JST), Hongo, Bunkyo-ku, Tokyo 113-0033, \\ Japan
}

\section{Supporting Information}

General. ${ }^{1} \mathrm{H}$ and ${ }^{13} \mathrm{C}$ NMR spectra were recorded on a JEOL JNM-LA300, JNM-LA400, or ECX-400 spectrometer in $\mathrm{CDCl}_{3}$ unless otherwise stored. Tetramethylsilane (TMS) was used as internal standard $(\delta=0)$ for ${ }^{1} \mathrm{H}$ NMR spectroscopy, and $\mathrm{CDCl}_{3}$ was used as internal standard $(\delta=77.0)$ for ${ }^{13} \mathrm{C}$ NMR spectroscopy. IR spectra were measured on a JASCO FT/IR-610 spectrometer. FAB-MS were measured with a JEOL JMS-MS700V. Commercially available chemicals and dry solvents (THF, $\mathrm{CH}_{2} \mathrm{Cl}_{2}, \mathrm{CH}_{3} \mathrm{CN}$, toluene, $\mathrm{CHCl}_{3}$ ), purchased from Aldrich, Kanto Chemical, Tokyo Chemical Industry and Wako Pure Chemical Industry, were purified according to standard procedures as required before use. Column chromatography was conducted on Silica gel 60 (Merck) and preparative thin-layer chromatography (PTLC) was carried out using Wakogel B-5F. The weight- and number-average molecular weights $\left(M_{\mathrm{w}}\right.$ and $M_{\mathrm{n}}$, respectively) and polydispersity indices $\left(M_{\mathrm{w}} / M_{\mathrm{n}}\right)$ of the linear copolymers were measured by gel permeation chromatography (GPC) at $40{ }^{\circ} \mathrm{C}$ using Shimadzu GPC system (system controller (SCL-10A VP), column oven (CTP-10A $V P)$, liquid chromatography (LC-10AD VP), degasser (DCV-12A), and refractive index detector (RID-10A)) equipped with a guard column (Shimadzu GPC-800P) and three mixed columns $\left(\right.$ Shimadzu GPC-8025 (exclusion limit $\left.=2 \times 10^{4}\right)$, GPC-803 (exclusion limit $\left.=7 \times 10^{4}\right)$, and GPC-804 (exclusion limit $\left.=4 \times 10^{5}\right)$ ). GPC was performed using THF as an eluent at a flow rate of $1 \mathrm{~mL} / \mathrm{min}$. Linear polystyrene standards were used for calibration. XRF and ICP analyses were performed using Shimadzu EDX-800 and Shimadzu ICPS-7510, respectively. Transmission electron microscopic (TEM) images were obtained using a JEOL JEM-1010 Electron Microscope operated at $80 \mathrm{kV}$. The details of TEM specimens were described below. The structures of known compounds were confirmed by comparison with data shown in literature. 
XRF analysis. The recovered crude compounds after the reaction were dissolved in THF and diluted to $5 \mathrm{~mL}$ with THF. The THF solution was used for XRF analysis to determine the Sc leaching. The solutions were recovered to isolate the desired compound after the analysis.

ICP analysis. The recovered crude compounds after the reaction were dissolved in $\mathrm{CH}_{3} \mathrm{CN}(1 \mathrm{~mL})$. The $\mathrm{CH}_{3} \mathrm{CN}$ solution was diluted to $10 \mathrm{~mL}$ with $\mathrm{CH}_{3} \mathrm{CN}$ and $5 \mathrm{~mL}$ of the solution was used for ICP analysis to determine the Sc leaching. The half of the solution was concentrated under reduced pressure, and the recovered crude compounds were treated to determine the yield of the desired compound. Corrected yield means double the isolated yield.

Preparation of copolymer 1. Styrene $\left(\begin{array}{lllll}8.33 & \mathrm{~g}, & 80.0 & \mathrm{mmol}\end{array}\right)$, 2-[(2-phenylallyloxy)methyl]oxirane ${ }^{1} \quad(1.90 \quad \mathrm{~g}, \quad 9.99 \mathrm{mmol}), \quad$ tetraethyleneglycol mono-2-phenyl-2-propenyl ether ${ }^{2}(3.10 \mathrm{~g}, 9.99 \mathrm{mmol})$, and AIBN $(84.0 \mathrm{mg}, 0.511 \mathrm{mmol})$ were mixed in $\mathrm{CHCl}_{3}(12 \mathrm{~mL})$. The mixture was stirred for $48 \mathrm{~h}$ at reflux and then cooled to room temperature. The resulting polymer solution was poured slowly onto $\mathrm{MeOH}$. The resulting white precipitated polymer was collected by filtration and washed with $\mathrm{MeOH}$ several times. The collected polymer was purified by repeated reprecipitation from the THF solution into $\mathrm{MeOH}$ and dried under reduced pressure to give copolymer 1 (9.00 g, 68\% yield). The molar ratio of the components was determined by ${ }^{1} \mathrm{H}$ NMR analysis $(\mathrm{x} / \mathrm{y} / \mathrm{z}=90 / 6 / 4) . \quad M_{\mathrm{w}}$ : 35019 , $M_{\mathrm{n}}: \quad 14524, M_{\mathrm{w}} / M_{\mathrm{n}}=2.41$.

Preparation of PI Sc(OTf) $)_{3}$. Copolymer $1(2.00 \mathrm{~g})$ was dissolved in toluene (40 mL) at room temperature, and to this solution was added $\mathrm{Sc}(\mathrm{OTf})_{3}(200 \mathrm{mg}, 0.406 \mathrm{mmol})$. The mixture was stirred for $20 \mathrm{~min}$ at this temperature. Hexane $(160 \mathrm{~mL})$ was slowly added to the mixture at room temperature. Coacervates were found to envelope $\operatorname{Sc}(\mathrm{OTf})_{3}$ in the media. The resulting catalyst capsules were collected by filtration after additional stirring for $30 \mathrm{~min}$ at room temperature and washed with hexane several times, then dried under reduced pressure. Next, the collected capsules were left at $120{ }^{\circ} \mathrm{C}$ for $2 \mathrm{~h}$ under solvent-free conditions to cross-link the polymer chain. Finally, the cross-linked catalyst was washed with toluene and $\mathrm{CH}_{2} \mathrm{Cl}_{2}$ several times, and dried at room temperature under reduced pressure to give PI Sc(OTf $)_{3}$ $\left(2.08 \mathrm{~g}, 0.191 \mathrm{mmol} / \mathrm{g}\right.$ of $\mathrm{Sc}(\mathrm{OTf})_{3}$ was loaded $)$. The Sc leaching in all filtrates and washings 
was measured by XRF analysis to determine the catalyst loading.

Preparation of PMI $\operatorname{Sc}(\mathbf{O T f})_{3}$. Copolymer $\mathbf{1}\left(\begin{array}{lll}2.00 & \mathrm{~g}\end{array}\right)$ was dissolved in THF-cyclohexane $(1: 3,140 \mathrm{~mL})$ at room temperature, and to this solution was slowly added a THF-cyclohexane $(1: 3,20 \mathrm{~mL})$ solution of $\mathrm{Sc}(\mathrm{OTf})_{3}(200 \mathrm{mg}, 0.406 \mathrm{mmol})$. After stirring for $30 \mathrm{~min}$ at room temperature, hexane $(160 \mathrm{~mL})$ was slowly added and then the mixture was stirred for $1 \mathrm{~h}$ at room temperature. The precipitated catalyst capsules were collected by filtration and washed with hexane several times, and dried under reduced pressure. Next, the collected capsules were left at $120{ }^{\circ} \mathrm{C}$ for $2 \mathrm{~h}$ under solvent-free conditions to cross-link the polymer chain. Finally, the cross-linked catalyst was washed with toluene and $\mathrm{CH}_{2} \mathrm{Cl}_{2}$ several times, and dried at room temperature under reduced pressure to give $\mathrm{PMI} \mathrm{Sc}(\mathrm{OTf})_{3}(2.01 \mathrm{~g}$, $0.185 \mathrm{mmol} / \mathrm{g}$ of $\mathrm{Sc}(\mathrm{OTf})_{3}$ was loaded).

\section{Preparation of TEM specimens in microencapsulation of $\operatorname{Sc}(\mathrm{OTf})_{3}$ using toluene as a} solvent. Copolymer $1(100 \mathrm{mg})$ was dissolved in toluene $(2 \mathrm{~mL})$ at room temperature, and to this solution was added $\mathrm{Sc}(\mathrm{OTf})_{3}(10 \mathrm{mg}, 0.020 \mathrm{mmol})$. The mixture was stirred for $20 \mathrm{~min}$ at this temperature. The resulting solution of $100 \mu \mathrm{L}$ was diluted to $4 \mathrm{~mL}$ with toluene and the diluted solution was dropped on carbon-coated $\mathrm{Cu}$ grids. The specimens without staining were allowed to dry in air.

\section{Preparation of TEM specimens in microcapsulation of $\operatorname{Sc}(\mathrm{OTf})_{3}$ using} THF-cyclohexane as a solvent. Copolymer $1(100 \mathrm{mg})$ was dissolved in THF-cyclohexane $(1: 3,7 \mathrm{~mL})$ at room temperature, and to this solution was slowly added a THF-cyclohexane $(1: 3,1 \mathrm{~mL})$ solution of $\mathrm{Sc}(\mathrm{OTf})_{3}(10 \mathrm{mg}, 0.020 \mathrm{mmol})$. The mixture was stirred for $30 \mathrm{~min}$ at this temperature. The resulting solution of $100 \mu \mathrm{L}$ was diluted to $1 \mathrm{ml}$ with THF-cyclohexane (1:3) and the diluted solution was dropped on carbon-coated $\mathrm{Cu}$ grids. The specimens without staining were allowed to dry in air.

A typical procedure for Mukaiyama aldol reaction. To a suspension of PMI $\mathrm{Sc}(\mathrm{OTf})_{3}(135 \mathrm{mg}, 0.025 \mathrm{mmol}, 5 \mathrm{~mol} \%)$ in $\mathrm{CH}_{2} \mathrm{Cl}_{2}(3.0 \mathrm{~mL})$ were added a solution of benzaldehyde $(53 \mathrm{mg}, 0.50 \mathrm{mmol})$ in $\mathrm{CH}_{2} \mathrm{Cl}_{2}(1.0 \mathrm{~mL})$ and a solution of the ketene silyl acetal derived from methyl isobutyrate $(113 \mathrm{mg}, 0.65 \mathrm{mmol})$ in $\mathrm{CH}_{2} \mathrm{Cl}_{2}(1.0 \mathrm{~mL})$ at $0{ }^{\circ} \mathrm{C}$ under Ar. The mixture was stirred for $10 \mathrm{~min}$ at the same temperature and stirred for $3 \mathrm{~h}$ at room temperature. After filtration and washing $\left(\mathrm{CH}_{2} \mathrm{Cl}_{2}\right.$, hexane), the filtrate was concentrated 
under reduced pressure. The divided crude compounds (see: ICP analysis section) were treated with $1 \mathrm{~N} \mathrm{HCl} / \mathrm{THF}(1 / 10)$ at room temperature to hydrolyze the silyl ether. After usual work up, the crude compounds were purified by PTLC to give methyl 3-hydroxy-2,2-dimethyl-3-phenylpropionate (48 mg, 46\% (corrected yield; 92\%)). PMI $\mathrm{Sc}(\mathrm{OTf})_{3}$ was recovered quantitatively (>97\%) and reused, the 2nd run; $96 \%$, the 3rd run; $94 \%$. In the 2 nd and 3 rd run, all recovered crude compounds after the reaction were treated to determine the yield of the desired compound.

Table 5. Substrate Scope for Mukaiyama Aldol Reaction

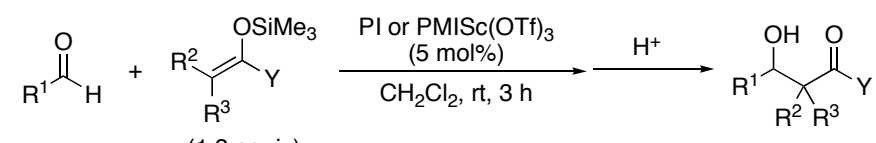

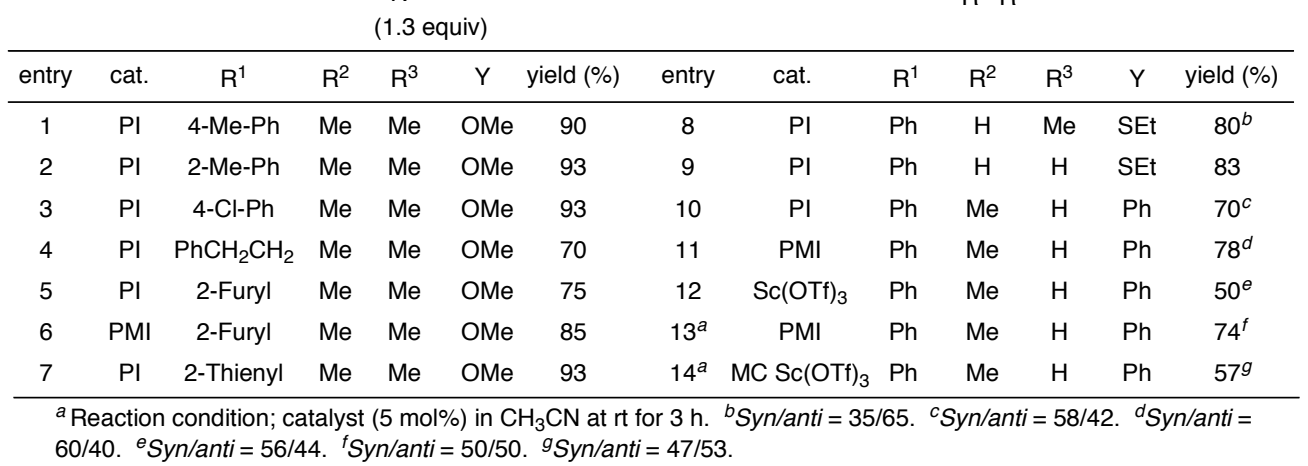

\section{Methyl 3-hydroxy-2,2-dimethyl-3-phenylpropionate: ${ }^{3}$}

White powder; ${ }^{1} \mathrm{H}$ NMR $\left(\mathrm{CDCl}_{3}\right) \delta=1.10$ (s, 3H), 1.14 (s, 3H), 3.03 (br s, $\left.1 \mathrm{H}\right), 3.70(\mathrm{~s}, 3 \mathrm{H})$, $4.88(\mathrm{~s}, 1 \mathrm{H}), 7.22-7.33(\mathrm{~m}, 5 \mathrm{H}) ;{ }^{13} \mathrm{C} \mathrm{NMR}\left(\mathrm{CDCl}_{3}\right) \delta=19.0,22.9,47.7,52.0,78.6,127,6$, $127.7,139.9,178.1$.

\section{Methyl 3-Hydroxy-2,2-dimethyl-3-(4-metylphenyl)propionate (entry 1):}

White powder; ${ }^{1} \mathrm{H}$ NMR $\left(\mathrm{CDCl}_{3}\right) \delta=1.09$ (s, 3H), 1.13 (s, 3H), 2.33 (s, 3H), 2.97 (br s, 1H), $3.71(\mathrm{~s}, 3 \mathrm{H}), 4.85$ (s, 3H), 7.10-7.19 (m, 4H); ${ }^{13} \mathrm{C} \mathrm{NMR}\left(\mathrm{CDCl}_{3}\right) \delta=19.0,21.1,23.0,47.7,52.0$, $78.5,127.5,128.4,137.0,137.3,178.2$.

\section{Methyl 3-hydroxy-2,2-dimethyl-3-(2-methylphenyl)propionate (entry 2):}

Colorless oil; ${ }^{1} \mathrm{H}$ NMR $\left(\mathrm{CDCl}_{3}\right) \delta=1.12$ (s, 3H), 1.20 (s, 3H), 2.37 (s, 3H), 2.96 (br s, 1H), 3.74 $(\mathrm{s}, 3 \mathrm{H}), 5.25(\mathrm{~s}, 1 \mathrm{H}), 7.10-7.22(\mathrm{~m}, 3 \mathrm{H}), 7.42(\mathrm{dd}, J=8.0$ and $2.0 \mathrm{~Hz}, 1 \mathrm{H}) ;{ }^{13} \mathrm{C} \mathrm{NMR}\left(\mathrm{CDCl}_{3}\right) \delta$ $=18.8,20.2,23.2,48.5,52.1,73.5,125.6,127.4,127.5,130.3,135.9,138.4,178.5$; IR (naet) 3498, 2952, $1725 \mathrm{~cm}^{-1}$; HRMS (FAB) Exact mass calcd for $\mathrm{C}_{13} \mathrm{H}_{19} \mathrm{O}_{3}[\mathrm{M}+\mathrm{H}]^{+}, 223.1334$. Found 223.1326. 
Methyl 3-hydroxy-2,2-dimethyl-3-(4-chrolophenyl)propionate (entry 3): ${ }^{3}$

White powder; ${ }^{1} \mathrm{H}$ NMR $\left(\mathrm{CDCl}_{3}\right) \delta=1.08(\mathrm{~s}, 3 \mathrm{H}), 1.12$ (s, 3H), $3.22(\mathrm{br} \mathrm{s}, 1 \mathrm{H}), 3.71(\mathrm{~s}, 3 \mathrm{H})$, $4.85(\mathrm{~s}, 3 \mathrm{H}), 7.21-7.25(\mathrm{~m}, 2 \mathrm{H}), 7.27-7.30(\mathrm{~m}, 2 \mathrm{H}) ;{ }^{13} \mathrm{C} \mathrm{NMR}\left(\mathrm{CDCl}_{3}\right) \delta=19.0,22.8,47.6$, $52.1,77.9,127.9,128.9,133.4,138.4,178.0$.

\section{Methyl 3-hydroxy-2,2-dimethyl-5-phenylpentanoate (entry 4): ${ }^{3}$}

Colorless oil; ${ }^{1} \mathrm{H}$ NMR $\left(\mathrm{CDCl}_{3}\right) \delta=1.16(\mathrm{~s}, 3 \mathrm{H}), 1.18$ (s, 3H), 1.55-1.67 (m, 1H), 1.70-1.81 (m, 1H), 2.56 (br s, 1H), 2.59-2.70 (m, 1H), 2.90-3.00 (m, 1H), 3.60-3.65 (m, 1H), 3.67 (s, 3H), 7.15-7.30 (m, 5H); ${ }^{13} \mathrm{C}$ NMR $\left(\mathrm{CDCl}_{3}\right) \delta=20.3,22.3,32.8,33.6,47.1,51.9,76.0,125.8,128.3$, $128.4,142.1,178.4$.

\section{Methyl 3-(2-furyl)-3-hydroxy-2,2-dimethylpropionate (entry 5): ${ }^{4}$}

Colorless oil; ${ }^{1} \mathrm{H}$ NMR $\left(\mathrm{CDCl}_{3}\right) \delta=1.20$ (s, 6H), 3.19 (br s, 1H), $3.72(\mathrm{~s}, 3 \mathrm{H}), 4.79$ (s, 1H), 6.23-6.27 (m, 1H), 6.31-6.34 (m, 1H), 7.33-7.36 (m, 1H); ${ }^{13} \mathrm{C} \mathrm{NMR}\left(\mathrm{CDCl}_{3}\right) \delta=20.1,22.7$, $47.1,52.1,73.3,107.7,110.0,141.7,153.9,177.6$.

\section{Methyl 3-hydroxy-2,2-dimethyl-3-(2-thienyl)propionate (entry 7):}

White powder; mp $45-46{ }^{\circ} \mathrm{C} ;{ }^{1} \mathrm{H}$ NMR $\left(\mathrm{CDCl}_{3}\right) \delta=1.20$ (s, 3H), $1.23(\mathrm{~s}, 3 \mathrm{H}), 3.17$ (br s, $\left.1 \mathrm{H}\right)$, $3.73(\mathrm{~s}, 3 \mathrm{H}), 5.10(\mathrm{~s}, 1 \mathrm{H}), 6.93-6.98(\mathrm{~m}, 2 \mathrm{H}), 7.23-7.27(\mathrm{~m}, 1 \mathrm{H}) ;{ }^{13} \mathrm{C} \mathrm{NMR}\left(\mathrm{CDCl}_{3}\right) \delta=19.9$, 22.7, 47.7, 52.1, 75.6, 124.8, 125.6, 126.2, 143.6, 177.9; IR (neat) 3475, 2982, $1722 \mathrm{~cm}^{-1}$; HRMS (FAB) Exact mass calcd for $\mathrm{C}_{10} \mathrm{H}_{13} \mathrm{O}_{2} \mathrm{~S}\left[\mathrm{M}-\mathrm{H}_{2} \mathrm{O}\right]^{+}$, 197.0636. Found 197.0640.

\section{$S$-Ethyl 3-hydroxy-2-methyl-3-phenylpropanetioate (entry 8): ${ }^{5}$}

Colorless oil; $($ syn/anti $=35 / 65): \quad{ }^{1} \mathrm{H}$ NMR $\left(\mathrm{CDCl}_{3}\right) \delta=0.92(\mathrm{~d}, J=7.2 \mathrm{~Hz}, 1.95 \mathrm{H}), 1.16(\mathrm{~d}, J$ $=6.8 \mathrm{~Hz}, 1.05 \mathrm{H}), 1.19(\mathrm{t}, J=7.2 \mathrm{~Hz}, 1.05 \mathrm{H}), 1.24(\mathrm{t}, J=7.2 \mathrm{~Hz}, 1.95 \mathrm{H}), 2.79-3.02(\mathrm{~m}, 4 \mathrm{H})$, $4.79(\mathrm{~d}, J=8.0 \mathrm{~Hz}, 0.65 \mathrm{H}), 5.07(\mathrm{~d}, J=3.6 \mathrm{~Hz}, 0.35 \mathrm{H}), 7.23-7.40(\mathrm{~m}, 5 \mathrm{H}),{ }^{13} \mathrm{C} \mathrm{NMR}\left(\mathrm{CDCl}_{3}\right) \delta$ $=11.4,14.5,15.3,23.1,23.2,54.9,55.4,73.7,76.5,126.0$, 126.6, 127.4, 128.0, 128.1, 128.4, 141.2, 141.6, 203.6, 203.9.

\section{$S$-Ethyl 3-hydroxy-3-phenylpropanetioate (entry 9): ${ }^{3}$}

Colorless oil; ${ }^{1} \mathrm{H}$ NMR $\left(\mathrm{CDCl}_{3}\right) \delta=1.25(\mathrm{t}, J=7.3 \mathrm{~Hz}, 3 \mathrm{H}), 2.86-3.01$ (m, 4H including q, $J=$ $7.3 \mathrm{~Hz}, 2 \mathrm{H}$ at 2.90), 3.13 (br s, $1 \mathrm{H}), 5.14-5.19(\mathrm{~m}, 1 \mathrm{H}), 7.25-7.37(\mathrm{~m}, 5 \mathrm{H}) ;{ }^{13} \mathrm{C} \mathrm{NMR}\left(\mathrm{CDCl}_{3}\right) \delta$ $=14.5,23.4,52.5,70.8,125.6,127.8,128.5,142.3,198.9$. 


\section{3-hydroxy-2-methyl-1,3-diphenyl-1-propanoe (entry 10): ${ }^{6}$}

Colorless oil; $($ syn/anti $=58 / 42): \quad{ }^{1} \mathrm{H}$ NMR $\left(\mathrm{CDCl}_{3}\right) \delta=1.04(\mathrm{~d}, J=7.2 \mathrm{~Hz}, 1.26 \mathrm{H}), 1.18(\mathrm{~d}, J$ $=7.2 \mathrm{~Hz}, 1.74 \mathrm{H}), 3.38(\mathrm{br}, 1 \mathrm{H}), 3.70(\mathrm{qd}, J=7.2$ and $3.2 \mathrm{~Hz}, 0.58 \mathrm{H}), 3.82(\mathrm{dq}, J=8.4$ and 7.2 $\mathrm{Hz}, 0.42 \mathrm{H}), 4.97(\mathrm{~d}, J=8.4 \mathrm{~Hz}, 0.42 \mathrm{H}), 5.21(\mathrm{~d}, J=3.2 \mathrm{~Hz}, 0.58 \mathrm{H}), 7.20-7.60(\mathrm{~m}, 8 \mathrm{H}), 7.91(\mathrm{~d}$, $J=7.2 \mathrm{~Hz}, 1.16 \mathrm{H}), 7.96(\mathrm{~d}, J=7.6 \mathrm{~Hz}, 0.84 \mathrm{H}) ;{ }^{13} \mathrm{C} \mathrm{NMR}\left(\mathrm{CDCl}_{3}\right) \delta=11.2,15.6,47.0,47.9$, $73.1,76.7,126.0,126.7,127.2,127.8,128.2$, 128.4, 128.6, 128.7, 133.2, 133.4, 135.6, 136.7, 141.8, 142.2, 204.8, 205.5.

A typical procedure for Mannich-type reaction. To a suspension of $\mathrm{PMI} \operatorname{Sc}(\mathrm{OTf})_{3}$ (136 mg, $0.025 \mathrm{mmol}, 10 \mathrm{~mol} \%)$ in $\mathrm{CH}_{3} \mathrm{CN}(3.0 \mathrm{~mL})$ were added a solution of N-benzylideneaniline $(45 \mathrm{mg}, 0.25 \mathrm{mmol})$ in $\mathrm{CH}_{3} \mathrm{CN}(1.0 \mathrm{~mL})$ and a solution of ketene silyl acetal derived from methyl isobutyrate $(65 \mathrm{mg}, 0.37 \mathrm{mmol})$ in $\mathrm{CH}_{3} \mathrm{CN}(1.0 \mathrm{~mL})$ at $0{ }^{\circ} \mathrm{C}$ under Ar. The mixture was stirred for $10 \mathrm{~min}$ at the same temperature and stirred for $3 \mathrm{~h}$ at room temperature. After filtration and washing $\left(\mathrm{CH}_{2} \mathrm{Cl}_{2}\right.$, hexane), the filtrate was concentrated under reduced pressure. The divided crude compounds (see: ICP analysis section) were purified by PTLC to give methyl 2,2-dimethyl-3-phenyl-3-( $N$-phenylamino)propionate (35 mg, $49 \%$ (corrected yield; 98\%)). PMI Sc(OTf $)_{3}$ was recovered quantitatively $(>97 \%)$, and reused, the 2nd run; 99\%, 3rd run; 97\%. In the 2nd and 3rd use, all recovered crude compounds after the reaction were treated to determine the yield of the desired compound.

Table 6. Substrate Scope for Mannich-type Reaction

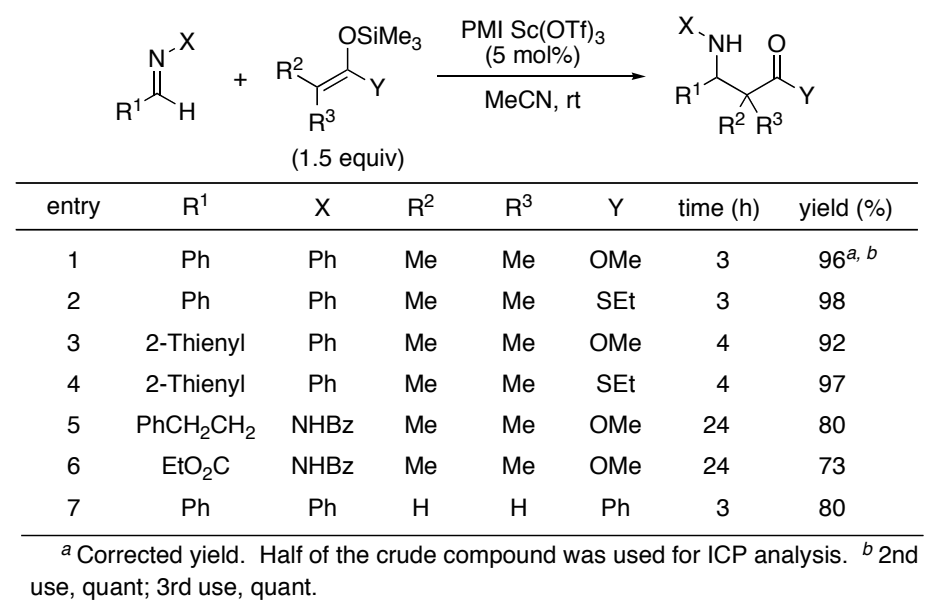

\section{Methyl 2,2-dimethyl-3-phenyl-3-( $N$-phenylamino)propionate (entry 1): ${ }^{5}$}

Pale brown powder; ${ }^{1} \mathrm{H}$ NMR $\left(\mathrm{CDCl}_{3}\right) \delta=1.16$ (s, 3H), 1.27 (s, 3H), $3.63(\mathrm{~s}, 3 \mathrm{H}), 4.49(\mathrm{~s}, 1 \mathrm{H})$, 4.84 (br s, 1H), 6.45-6.53 (m, 2H), 6.55-6.62 (m, 1H), 7.00-7.06 (m, 2H), 7.18-7.30 (m, 5H); 
${ }^{13} \mathrm{C}$ NMR $\left(\mathrm{CDCl}_{3}\right) \delta=20.7,24.5,46.9,52.0,64.4,113.4,117.3,127.4,127.9,128.2,129.0$, 139.1, 146.8, 177.0.

\section{$S$-Ethyl 2,2-dimethyl-3-phenyl-3-( $N$-phenylamino)propanethioate (entry 2):}

Gray powder; mp $70-71{ }^{\circ} \mathrm{C} ;{ }^{1} \mathrm{H}$ NMR $\left(\mathrm{CDCl}_{3}\right) \delta=1.17(\mathrm{t}, J=8.0 \mathrm{~Hz}, 3 \mathrm{H}), 1.23(\mathrm{~s}, 3 \mathrm{H}), 1.25(\mathrm{~s}$, $3 \mathrm{H}), 2.83(\mathrm{~m}, 2 \mathrm{H}), 4.51(\mathrm{~s}, 1 \mathrm{H}), 4.80(\mathrm{br} \mathrm{s}, 1 \mathrm{H}), 6.45-6.50(\mathrm{~m}, 2 \mathrm{H}), 6.55-6.60(\mathrm{~m}, 1 \mathrm{H})$, 7.01-7.04 (m, 2H), 7.20-7.31 (m, 5H); ${ }^{13} \mathrm{C} \mathrm{NMR}\left(\mathrm{CDCl}_{3}\right) \delta=14.4,19.9,23.4,25.1,53.2,64.9$, 113.3, 117.2, 127.4, 127.9, 128.5, 128.9, 139.0, 146.9, 206.1; IR (neat) 3409, 2972, 1665, 1601 $\mathrm{cm}^{-1}$; HRMS (FAB) Exact mass calcd for $\mathrm{C}_{19} \mathrm{H}_{24} \mathrm{NOS}[\mathrm{M}+\mathrm{H}]^{+}, 314.1579$. Found 314.1574.

\section{Methyl 2,2-dimethyl-3-( $N$-phenylamino)-3-(2-thienyl)propionate (entry 3): ${ }^{7}$}

Pale brown powder; ${ }^{1} \mathrm{H}$ NMR $\left(\mathrm{CDCl}_{3}\right) \delta=1.27$ (s, 3H), 1.32 (s, 3H), 3.67 (s, 3H), 4.70 (br s, $1 \mathrm{H}), 4.82(\mathrm{~s}, 1 \mathrm{H}), 6.50-6.70(\mathrm{~m}, 3 \mathrm{H}), 6.86-6.96(\mathrm{~m}, 2 \mathrm{H}), 7.04-7.16(\mathrm{~m}, 3 \mathrm{H}) ;{ }^{13} \mathrm{C} \mathrm{NMR}\left(\mathrm{CDCl}_{3}\right)$ $\delta=21.4,23.9,47.3,52.1,60.6,113.6,117.9,124.4,126.0,126.4,129.0,144.0,146.7,176.8$.

\section{$S$-Ethyl 2,2-dimethyl-3-( $N$-phenylamino)-3-(2-thienyl)propanethioate (entry 4):}

Pale brown powder; mp $65-66{ }^{\circ} \mathrm{C} ;{ }^{1} \mathrm{H}$ NMR $\left(\mathrm{CDCl}_{3}\right) \delta=1.18(\mathrm{t}, J=7.6 \mathrm{~Hz}, 3 \mathrm{H}), 1.32(\mathrm{~s}, 3 \mathrm{H})$, $1.34(\mathrm{~s}, 3 \mathrm{H}), 2.84(\mathrm{q}, J=7.6 \mathrm{~Hz}, 2 \mathrm{H}), 4.67(\mathrm{br} \mathrm{s}, 1 \mathrm{H}), 4.84(\mathrm{~s}, 1 \mathrm{H}), 6.52-6.60(\mathrm{~m}, 2 \mathrm{H})$, 6.62-6.68 (m, 1H), 6.88-6.96 (m, 2H), 7.03-7.10 (m, 2H), 7.12-7.16 (m, 1H); ${ }^{13} \mathrm{C}$ NMR $\left(\mathrm{CDCl}_{3}\right) \delta=14.4,20.8,23.4,24.6,53.5,61.2,113.5,117.9,124.5,126.1,126.4,129.0,144.0$, 146.8, 205.9; IR (neat) 3403, 2972, 1664, $1601 \mathrm{~cm}^{-1}$; HRMS (FAB) Exact mass calcd for $\mathrm{C}_{17} \mathrm{H}_{22} \mathrm{NOS}_{2}[\mathrm{M}+\mathrm{H}]^{+}, 320.1143$. Found 320.1153 .

\section{Methyl 3-( $N$ '-benzoylhydrazino)-2,2-dimethyl-5-phenylpentanoate (entry 5): ${ }^{8}$}

Pale yellow oil; ${ }^{1} \mathrm{H}$ NMR $\left(\mathrm{CDCl}_{3}\right) \delta=1.18(\mathrm{~s}, 3 \mathrm{H}), 1.25$ (s, 3H), 1.62-1.82 (m, 2H), 2.72-2.82 (m, 1H), 2.98-3.08 (m, 1H), $3.18(\mathrm{dd}, J=9.6$ and $2.4 \mathrm{~Hz}, 1 \mathrm{H}), 3.57(\mathrm{~s}, 3 \mathrm{H}), 4.80(\mathrm{br} \mathrm{s}, 1 \mathrm{H})$, 7.10-7.27 (m, 5H), 7.35-7.54 (m, 3H), 7.74-7.76 (m, 2H), 7.89 (br s, $1 \mathrm{H}) ;{ }^{13} \mathrm{C} \mathrm{NMR}\left(\mathrm{CDCl}_{3}\right) \delta$ $=20.5,23.1,31.1,33.5,46.7,51.9,66.4,125.8,126.7,128.3,128.4,128.6,131.7,132.5,141.8$, 166.1, 178.7 .

\section{1-Methyl Ethyl 3-( $N$ '-benzoylhydrazino)-2,2-dimethylsuccinate (entry 6): ${ }^{9}$}

Colorless powder; ${ }^{1} \mathrm{H}$ NMR $\left(\mathrm{CDCl}_{3}\right) \delta=1.25(\mathrm{~s}, 3 \mathrm{H}), 1.26(\mathrm{t}, J=7.2 \mathrm{~Hz}, 3 \mathrm{H}), 1.36(\mathrm{~s}, 3 \mathrm{H}), 3.72$ (s, 3H), $3.95(\mathrm{~s}, 1 \mathrm{H}), 4.14-4.28(\mathrm{~m}, 2 \mathrm{H}), 5.35(\mathrm{br} \mathrm{s}, 1 \mathrm{H}), 7.39-7.44(\mathrm{~m}, 2 \mathrm{H}), 7.48-7.53(\mathrm{~m}, 1 \mathrm{H})$, 
$7.74(\mathrm{~d}, J=7.2 \mathrm{~Hz}, 2 \mathrm{H}), 8.05($ br s, $1 \mathrm{H}) ;{ }^{13} \mathrm{C} \mathrm{NMR}\left(\mathrm{CDCl}_{3}\right) \delta=14.0,21.7,21.9,45.6,52.1,61.3$, $69.3,126.9,128.6,131.8,132.5,167.0,171.2,176.1$.

\section{1,3-Diphenyl-3-( $N$-phenylamino)propanone (entry 7): ${ }^{5}$}

Pale yellow powder; ${ }^{1} \mathrm{H}$ NMR $\left(\mathrm{CDCl}_{3}\right) \delta=3.44(\mathrm{dd}, J=16.1$ and $7.4 \mathrm{~Hz}, 1 \mathrm{H}), 3.52(\mathrm{dd}, J=$ 16.1 and $5.6 \mathrm{~Hz}, 1 \mathrm{H}), 4.85$ (br s, $1 \mathrm{H}), 5.00(\mathrm{dd}, J=7.4$ and $5.6 \mathrm{~Hz}, 1 \mathrm{H}), 6.54-6.61(\mathrm{~m}, 2 \mathrm{H})$, 6.64-6.70 (m, 1H), 7.05-7.13 (m, 2H), 7.20-7.25 (m, 1H), 7.28-7.35 (m, 2H), 7.38-7.48 (m, $4 \mathrm{H}), 7.52-7.58(\mathrm{~m}, 1 \mathrm{H}), 7.88-7.92(\mathrm{~m}, 2 \mathrm{H}) ;{ }^{13} \mathrm{C} \mathrm{NMR}\left(\mathrm{CDCl}_{3}\right) \delta=46.2,55.0,114.0,118.0$, $126.4,127.4,128.2,128.7,128.8,129.1,133.4,136.6,142.7,146.6,198.2$.

A typical procedure for Michael reaction. To a suspension of PMI Sc(OTf $)_{3}(109 \mathrm{mg}$, $0.020 \mathrm{mmol}, 5 \mathrm{~mol} \%)$ in $\mathrm{CH}_{3} \mathrm{CN}(4.0 \mathrm{~mL})$ were added a solution of methyl 1-oxoindan-2-carboxylate $(76 \mathrm{mg}, 0.40 \mathrm{mmol})$ in $\mathrm{CH}_{3} \mathrm{CN}(1.0 \mathrm{~mL})$ and methyl vinyl ketone (67 $\mu \mathrm{L}, 0.80 \mathrm{mmol}$ ) at $0{ }^{\circ} \mathrm{C}$ under Ar. The mixture was stirred for $10 \mathrm{~min}$ at the same temperature and stirred for $4 \mathrm{~h}$ at room temperature. After filtration and washing $\left(\mathrm{CH}_{2} \mathrm{Cl}_{2}\right.$, hexane), the filtrate was concentrated under reduced pressure. The divided crude compounds (see: ICP analysis section) were purified by PTLC to give methyl 1-oxo-2-(3-oxo-butyl)indan-2-carboxylate (48 mg, 46\% (corrected yield; 92\%)). PMI $\mathrm{Sc}(\mathrm{OTf})_{3}$ was recovered quantitatively (> 99\%), and reused, the 2nd run; $94 \%$, 3rd run; $92 \%$. In the 2 nd and 3 rd use, all recovered crude compounds after the reaction were treated to determine the yield of the desired compound.

Table 7. Michael Reaction Using PMI Sc(OTf $)_{3}$ in Other Solvents

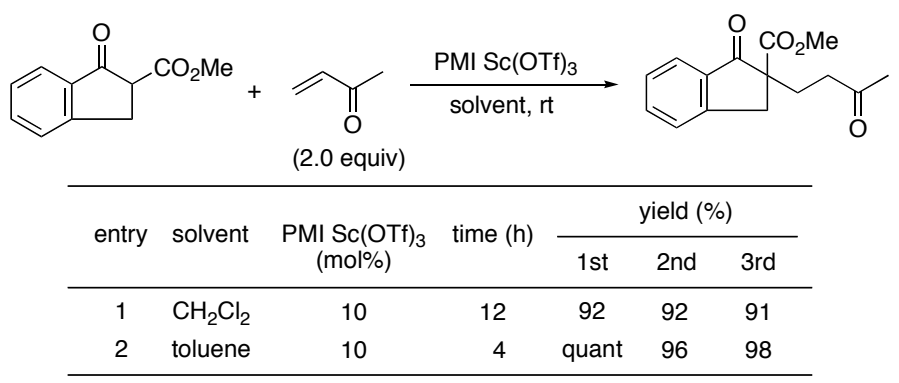


Table 8. Substrate Scope for Michael Reaction

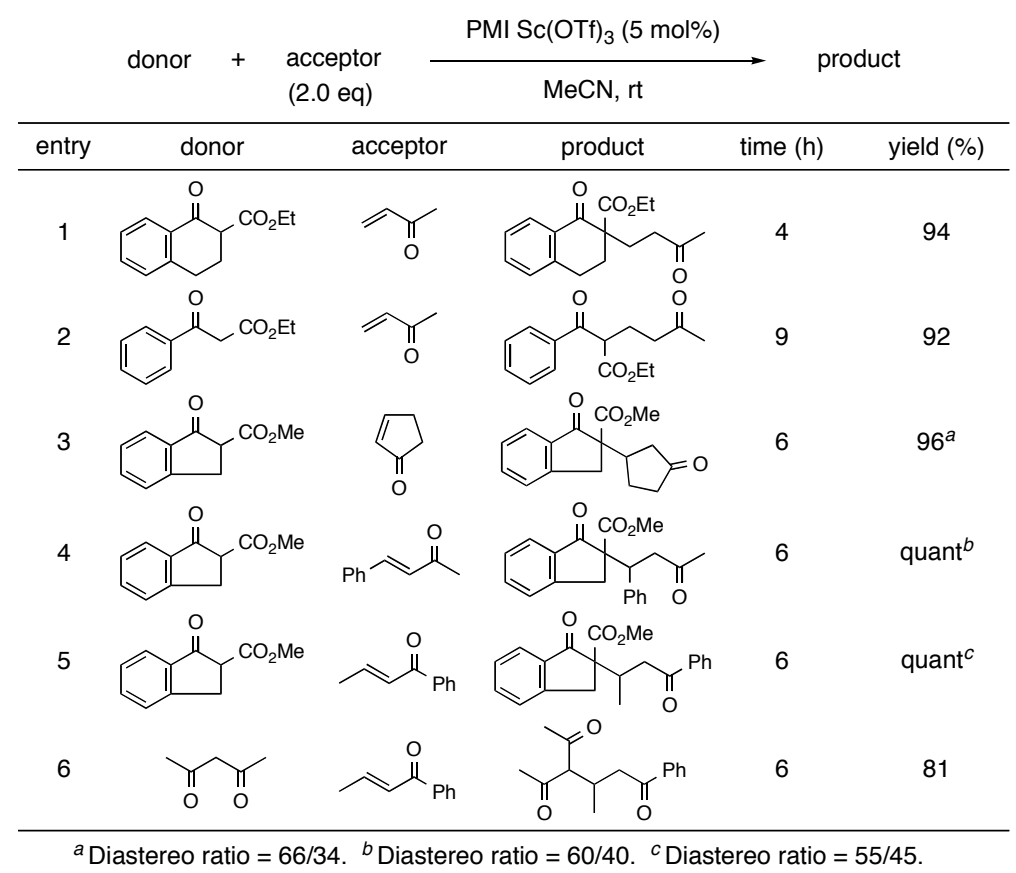

\section{Methyl 1-oxo-2-(3-oxo-butyl)indan-2-carboxylate (Table 7): ${ }^{10}$}

White powder; ${ }^{1} \mathrm{H}$ NMR $\left(\mathrm{CDCl}_{3}\right) \delta=2.12(\mathrm{~s}, 3 \mathrm{H}), 2.18-2.30(\mathrm{~m}, 2 \mathrm{H}), 2.51(\mathrm{ddd}, J=15.2,9.6$ and $5.6 \mathrm{~Hz}, 1 \mathrm{H}), 2.63(\mathrm{ddd}, J=15.2,10.0$ and $6.0 \mathrm{~Hz}, 1 \mathrm{H}), 3.05(\mathrm{~d}, J=17.6 \mathrm{~Hz}, 1 \mathrm{H}), 3.68(\mathrm{~d}, J$ = 17.6 Hz, 1H), $3.69(\mathrm{~s}, 3 \mathrm{H}), 7.39-7.44(\mathrm{~m}, 1 \mathrm{H}), 7.46-7.49(\mathrm{~m}, 1 \mathrm{H}), 7.62-7.66(\mathrm{~m}, 1 \mathrm{H})$, 7.76-7.79 $(\mathrm{m}, 1 \mathrm{H}) ;{ }^{13} \mathrm{C} \mathrm{NMR}\left(\mathrm{CDCl}_{3}\right) \delta=28.5,29.9,37.8,38.7,52.7,59.0,124.8,126.4,127.9$, 135.0, 135.5, 152.5, 171.5, 202.2, 207.3.

Ethyl 1-oxo-2-(3-oxo-butyl)-1,2,3,4-tetrahydronaphtalene-2-carboxylate (Table 8, entry 1): ${ }^{11}$

Colorless oil; ${ }^{1} \mathrm{H}$ NMR $\left(\mathrm{CDCl}_{3}\right) \delta=1.17(\mathrm{t}, J=7.2 \mathrm{~Hz}, 3 \mathrm{H}), 2.05-2.28(\mathrm{~m}, 3 \mathrm{H}), 2.15(\mathrm{~s}, 3 \mathrm{H})$, 2.50-2.63 (m, 2H), 2.66-2.78 (m, 1H), 2.90-3.10 (m, 2H), 4.15 (d, $J=7.2 \mathrm{~Hz}, 2 \mathrm{H}), 7.18-7.24$ (m, 1H), 7.27-7.35 (m, 1H), 7.43-7.50 (m, 1H), 8.00-8.05 (m, 1H); ${ }^{13} \mathrm{C} \mathrm{NMR}\left(\mathrm{CDCl}_{3}\right) \delta=14.0$, 25.8, 27.5, 29.9, 31.6, 39.0, 56.5, 61.3, 126.8, 127.8, 128.6, 131.9, 133.5, 142.7, 171.8, 195.5, 207.7.

\section{Ethyl 2-benzoyl-5-oxo-hexanoate (entry 2): ${ }^{11}$}

Colorless oil; ${ }^{1} \mathrm{H}$ NMR $\left(\mathrm{CDCl}_{3}\right) \delta=1.17(\mathrm{t}, J=7.2 \mathrm{~Hz}, 3 \mathrm{H}), 2.14(\mathrm{~s}, 3 \mathrm{H}), 2.16-2.32(\mathrm{~m}, 2 \mathrm{H})$, 2.51-2.68 (m, 2H), 4.10-4.19 (m, 2H), $4.45(\mathrm{dd}, J=6.4$ and $6.3 \mathrm{~Hz}, 1 \mathrm{H}), 7.45-7.52(\mathrm{~m}, 2 \mathrm{H})$, 
7.55-7.62 (m. 1H), 8.00-8.05 (m, 2H); ${ }^{13} \mathrm{C} \mathrm{NMR}\left(\mathrm{CDCl}_{3}\right) \delta=13.9,22.6,29.9,40.4,52.6,61.3$, 128.6, 128.7, 133.5, 135.9, 169.7, 195.2, 207.7.

\section{Methyl 1-oxo-2-(3-oxo-cyclopentyl)indan-2-carboxylate (entry 3):}

A mixture of the two diastereomers $(\mathrm{dr}=66 / 34)$; colorless oil; ${ }^{1} \mathrm{H}$ NMR $\left(\mathrm{CDCl}_{3}\right) \delta=1.23-2.62$ (m, 6H), $3.15(\mathrm{~d}, J=17.8 \mathrm{~Hz}, 1 \mathrm{H}), 3.18-3.30(\mathrm{~m}, 1 \mathrm{H}), 3.70(\mathrm{~s}, 1.02 \mathrm{H}), 3.72(\mathrm{~s}, 1.98 \mathrm{H}), 3.73(\mathrm{~d}$, $J=17.8 \mathrm{~Hz}, 1 \mathrm{H}), 7.39-7.46(\mathrm{~m}, 1 \mathrm{H}), 7.49-7.56(\mathrm{~m}, 1 \mathrm{H}), 7.60-7.70(\mathrm{~m}, 1 \mathrm{H}), 7.74-7.82(\mathrm{~m}$, $1 \mathrm{H}) ;{ }^{13} \mathrm{C} \mathrm{NMR}\left(\mathrm{CDCl}_{3}\right) \delta=23.9,25.2,33.4,33.8,38.07,38.14,39.3,40.5,40.7,40.8,52.8$, $62.0,62.4,124.7,124.8,126.2,126.3,128.01,128.04,135.2,135.4,135.7,152.7,152.9,170.6$, 170.7, 201.27, 201.33, 216.7, 216.9; IR (neat) 2954, 1743, 1708, $1609 \mathrm{~cm}^{-1}$; HRMS (FAB) Exact mass calcd for $\mathrm{C}_{16} \mathrm{H}_{17} \mathrm{O}_{4}[\mathrm{M}+\mathrm{H}]^{+}, 273.1127$. Found 273.1128 .

\section{Methyl 1-oxo-2-(1-phenyl-3-oxo-butyl)indan-2-carboxylate (entry 4):}

Major: Waxy solid; ${ }^{1} \mathrm{H}$ NMR $\left(\mathrm{CDCl}_{3}\right) \delta=2.00(\mathrm{~s}, 3 \mathrm{H}), 2.81(\mathrm{dd}, J=16.5$ and $3.0 \mathrm{~Hz}, 1 \mathrm{H})$, $3.11(\mathrm{dd}, J=16.5$ and $11.0 \mathrm{~Hz}, 1 \mathrm{H}), 3.28(\mathrm{~d}, J=17.4 \mathrm{~Hz}, 1 \mathrm{H}), 3.62(\mathrm{~d}, J=17.4 \mathrm{~Hz}, 1 \mathrm{H}), 3.63(\mathrm{~s}$, $3 \mathrm{H}), 4.35(\mathrm{dd}, J=11.0$ and $3.0 \mathrm{~Hz}, 1 \mathrm{H}), 7.18-7.23(\mathrm{~m}, 5 \mathrm{H}), 7.32-7.37(\mathrm{~m}, 2 \mathrm{H}), 7.51-7.56(\mathrm{~m}$, 1H), 7.73-7.77 (m, 1H); ${ }^{13} \mathrm{C} \mathrm{NMR}\left(\mathrm{CDCl}_{3}\right) \delta=30.1,35.0,44.6,45.3,52.8,64.6,124.4,126.1$, 127.3, 127.7, 128.3 , 128.8, 135.4, 136.0, 138.9, 152.8, 170.6, 201.8, 205.8; IR (neat) 2955, 1712, $1605 \mathrm{~cm}^{-1}$; HRMS (FAB) Exact mass calcd for $\mathrm{C}_{21} \mathrm{H}_{21} \mathrm{O}_{4}[\mathrm{M}+\mathrm{H}]^{+}, 337.1440$. Found 337.1439.

Minor: Pale yellow powder; mp $160{ }^{\circ} \mathrm{C}$ (decomp.); ${ }^{1} \mathrm{H}$ NMR $\left(\mathrm{CDCl}_{3}\right) \delta=2.06(\mathrm{~s}, 3 \mathrm{H})$, 3.12-3.17 (m, 2H), 3.29 (d, $J=17.4 \mathrm{~Hz}, 1 \mathrm{H}), 3.72$ (s, 3H), 3.73 (d, $J=17.4 \mathrm{~Hz}, 1 \mathrm{H}), 4.29$ (dd, $J$ $=8.7$ and $6.0 \mathrm{~Hz}, 1 \mathrm{H}), 7.03-7.27(\mathrm{~m}, 5 \mathrm{H}), 7.32-7.36(\mathrm{~m}, 1 \mathrm{H}), 7.46-7.52(\mathrm{~m}, 1 \mathrm{H}), 7.54-7.58(\mathrm{~m}$, $1 \mathrm{H}) ;{ }^{13} \mathrm{C} \mathrm{NMR}\left(\mathrm{CDCl}_{3}\right) \delta=29.9,33.8,44.2,45.6,52.9,64.8,124.7,125.9,127.3,127.6,128.2$, 129.3, 134.7, 135.3, 138.2, 152.8, 170.5, 200.7, 206.3; IR (neat) 2933, 1711, $1644 \mathrm{~cm}^{-1}$; HRMS (FAB) Exact mass calcd for $\mathrm{C}_{21} \mathrm{H}_{21} \mathrm{O}_{4}[\mathrm{M}+\mathrm{H}]^{+}, 337.1440$. Found 337.1432.

\section{Methyl 1-oxo-2-(1-methyl-3-oxo-3-phenylpropyl)indan-2-carboxylate (entry 5):}

A mixture of the two diastereomers $(\mathrm{dr}=55 / 45)$; colorless oil; ${ }^{1} \mathrm{H}$ NMR $\left(\mathrm{CDCl}_{3}\right) \delta=0.82(\mathrm{~d}, J$ $=6.9 \mathrm{~Hz}, 1.65 \mathrm{H}), 0.96(\mathrm{~d}, J=6.9 \mathrm{~Hz}, 1.35 \mathrm{H}), 2.69(\mathrm{dd}, J=15.4$ and $11.2 \mathrm{~Hz}, 0.45 \mathrm{H}), 2.79(\mathrm{dd}$, $J=15.1$ and $10.5 \mathrm{~Hz}, 0.55 \mathrm{H}), 3.00(\mathrm{dd}, J=15.1$ and $2.3 \mathrm{~Hz}, 0.55 \mathrm{H}), 3.20(\mathrm{~d}, J=17.9 \mathrm{~Hz}$, $0.45 \mathrm{H}), 3.22(\mathrm{~d}, J=17.6 \mathrm{~Hz}, 0.55 \mathrm{H}), 3.26-3.45(\mathrm{~m}, 1.45 \mathrm{H}), 3.70(\mathrm{~s}, 3 \mathrm{H}), 3.77(\mathrm{~d}, J=17.9 \mathrm{~Hz}$, $0.45 \mathrm{H}), 3.78(\mathrm{~d}, J=17.6 \mathrm{~Hz}, 0.55 \mathrm{H}), 7.35-7.59(\mathrm{~m}, 5 \mathrm{H}), 7.60-7.68(\mathrm{~m}, 1 \mathrm{H}) 7.72-7.81(\mathrm{~m}, 1 \mathrm{H})$, 7.90-7.96 (m, 1H), 7.99-8.05 (m, 1H); ${ }^{13} \mathrm{C} \mathrm{NMR}\left(\mathrm{CDCl}_{3}\right) \delta=14.8,15.6,33.0,33.9,34.0,40.9$, 
$42.1,52.8,52.9,64.7,65.0,124.5,124.6,126.2,126.3,127.76,127.82,128.2,128.6,133.0$, 133.1, 135.42, 135.45, 135.6, 135.8, 136.6, 136.7, 153.2, 153.3, 170.8, 198.6, 198.8, 201.6, 202.0; IR (neat) 2958, 1742, 1713, $1606 \mathrm{~cm}^{-1}$; HRMS (FAB) Exact mass calcd for $\mathrm{C}_{21} \mathrm{H}_{21} \mathrm{O}_{4}$ $[\mathrm{M}+\mathrm{H}]^{+}, 337.1440$. Found 337.1439.

\section{4-Acetyl-3-methyl-1-phenylhexane-1,5-dione (entry 6):}

Colorless oil; ${ }^{1} \mathrm{H}$ NMR $\left(\mathrm{CDCl}_{3}\right) \delta=1.00(\mathrm{~d}, J=6.4 \mathrm{~Hz}, 3 \mathrm{H}), 2.20(\mathrm{~s}, 3 \mathrm{H}), 2.23(\mathrm{~s}, 3 \mathrm{H})$, 2.80-2.89 (m, 1H), 2.96-3.12 (m, 2H), $3.83(\mathrm{~d}, J=8.7 \mathrm{~Hz}, 1 \mathrm{H}), 7.42-7.52(\mathrm{~m}, 2 \mathrm{H}), 7.53-7.60$ $(\mathrm{m}, 1 \mathrm{H}), 7.88-8.02(\mathrm{~m}, 2 \mathrm{H}) ;{ }^{13} \mathrm{C} \mathrm{NMR}\left(\mathrm{CDCl}_{3}\right) \delta=17.7,29.7,29.8,30.2,42.5,73.6,128.0$, 128.6, 133.2, 136.8, 198.8, 204.0, 204.3; IR (neat) 2968, 1692, $1597 \mathrm{~cm}^{-1}$; HRMS (FAB) Exact mass calcd for $\mathrm{C}_{15} \mathrm{H}_{19} \mathrm{O}_{3}[\mathrm{M}+\mathrm{H}]^{+}, 247.1334$. Found 247.1330.

Control experiments. PI Sc $(\mathrm{OTf})_{3}(2)$ was prepared using copolymer 2, an analogue of copolymer $\mathbf{1}$, in which the tetraethyleneglycol moieties were replaced by the isometric alkyl alcohol moieties. PI $\mathrm{Sc}(\mathrm{OTf})_{3}(2)$ was evaluated in the Mukaiyama aldol reaction. It was found that $12.6 \%$ of Sc was leached, although a comparable catalytic activity with that of PI $\mathrm{Sc}(\mathrm{OTf})_{3}$ prepared using copolymer 1 was observed. Moreover, PI Sc(OTf $)_{3}$ (2) could not be prepared using toluene as a solvent, because coaceravation did not occur. These results strongly suggested that the tetramethyleneglycol moieties in copolymer $\mathbf{1}$ played an important role in the immobilization of $\mathrm{Sc}(\mathrm{OTf})_{3}$.

Scheme 1. Control Experiments

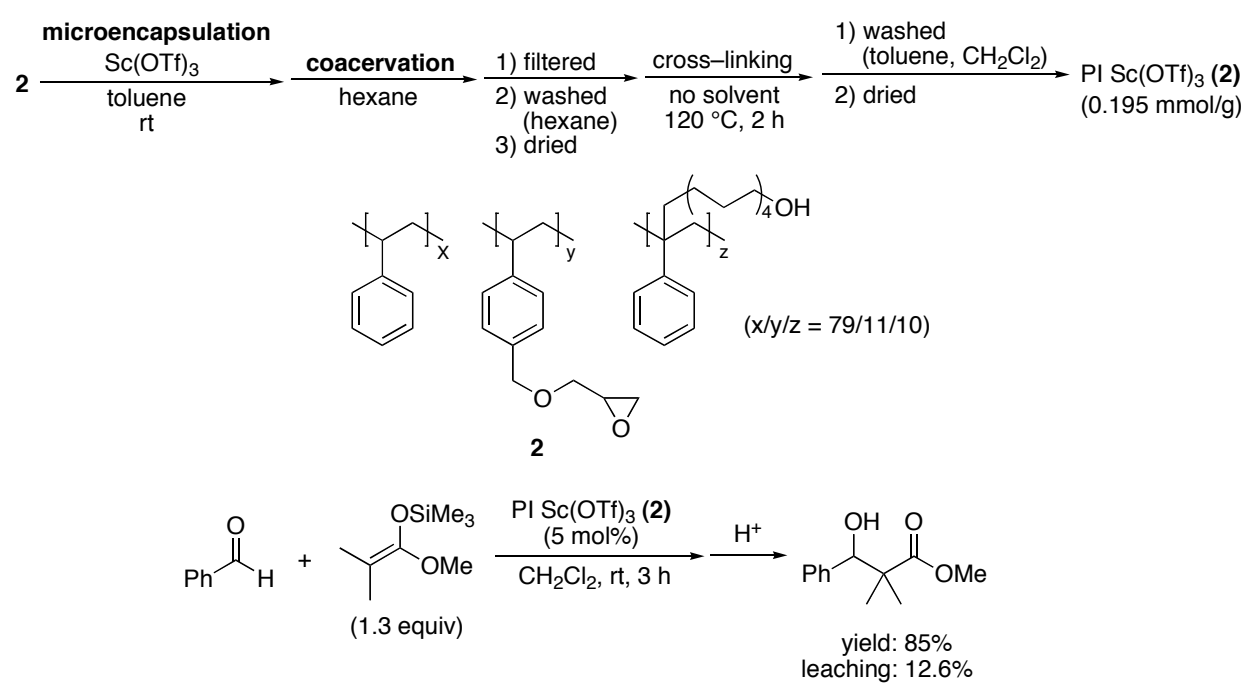

Preparation of copolymer 2. Styrene (9.08 g, $87.2 \mathrm{mmol})$, 4-vinylbenzyl glycidyl ether $^{2}(2.07 \mathrm{~g}, 10.9 \mathrm{mmol}), 14-$ phenylpentadec-14-en-1-ol ${ }^{12}$ (3.30 g, $\left.10.9 \mathrm{mmol}\right)$, and AIBN 
(129 mg, $0.785 \mathrm{mmol})$ were mixed in $\mathrm{CHCl}_{3}(13 \mathrm{~mL})$. The mixture was stirred for $24 \mathrm{~h}$ at reflux and then cooled to room temperature. The resulting polymer solution was poured slowly onto $\mathrm{MeOH}$. The resulting white precipitated polymer was collected by filtration and washed with $\mathrm{MeOH}$ several times. The collected polymer was purified by repeated reprecipitation from the THF solution into $\mathrm{MeOH}$ and dried under reduced pressure to give copolymer 2 (6.95 g, 48\% yield). The molar ratio of the components was determined by ${ }^{1} \mathrm{H}$ NMR analysis $(\mathrm{x} / \mathrm{y} / \mathrm{z}=79 / 11 / 10) . \quad M_{\mathrm{w}}: \quad 23843, M_{\mathrm{n}}: \quad 18857, M_{\mathrm{w}} / M_{\mathrm{n}}=1.26$.

Preparation of PI Sc(OTf) $)_{3}$ (2). Copolymer $2(500 \mathrm{mg})$ was dissolved in $\mathrm{CH}_{2} \mathrm{Cl}_{2}(10$ $\mathrm{mL})$ at room temperature, and to this solution was added $\mathrm{Sc}(\mathrm{OTf})_{3}(50 \mathrm{mg}, 0.10 \mathrm{mmol})$. The mixture was stirred for $20 \mathrm{~min}$ at this temperature. Hexane $(40 \mathrm{~mL})$ was slowly added to the mixture at room temperature. Coacervates were found to envelope $\operatorname{Sc}(\mathrm{OTf})_{3}$ in the media. The resulting catalyst capsules were collected by filtration after additional stirring for $30 \mathrm{~min}$ at room temperature and washed with hexane several times, then dried under reduced pressure. Next, the collected capsules were left at $120{ }^{\circ} \mathrm{C}$ for $2 \mathrm{~h}$ under solvent-free conditions to cross-link the polymer chain. Finally, the cross-linked catalyst was washed with toluene and $\mathrm{CH}_{2} \mathrm{Cl}_{2}$ several times, and dried at room temperature under reduced pressure to give PI Sc(OTf $)_{3}$ $\left(527 \mathrm{mg}, 0.195 \mathrm{mmol} / \mathrm{g}\right.$ of $\mathrm{Sc}(\mathrm{OTf})_{3}$ was loaded).

Scheme 2. Hydroxmethylation with Formaldehyde Water Solution Using PMI Sc(OTf $)_{3}$

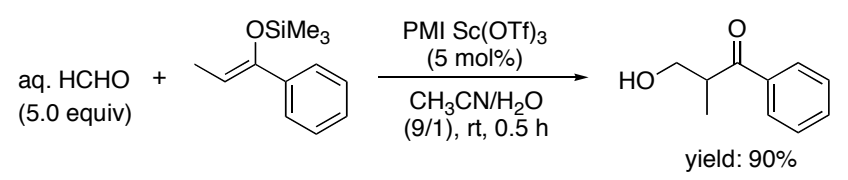

Hydroxymethylation of the silyl enol ether of propiophenone with a formaldehyde water solution. To a suspension of PMI Sc(OTf $)_{3}(109 \mathrm{mg}, 0.020 \mathrm{mmol}, 5 \mathrm{~mol} \%)$ in $\mathrm{CH}_{3} \mathrm{CN}$ $(1.85 \mathrm{~mL})$ were added a $37 \%$ formaldehyde water solution $(150 \mu \mathrm{L}, 201 \mathrm{mmol})$ and the silyl enol ether of propiophene ( $84 \mathrm{mg}, 0.41 \mathrm{mmol})$ at room temperature. The mixture was stirred for $0.5 \mathrm{~h}$ at room temperature. After filtration and washing $\left(\mathrm{CH}_{2} \mathrm{Cl}_{2}\right.$, hexane), the filtrate was concentrated under reduced pressure, and the resulting crude compound was purified by PTLC to give 3-hydroxy-2-methyl-1-phenylpropane-1-onemethyl ${ }^{14}$ (60 mg, 90\%). Colorless oil; ${ }^{1} \mathrm{H}$ $\operatorname{NMR}\left(\mathrm{CDCl}_{3}\right) \delta=1.25(\mathrm{~d}, J=6.9 \mathrm{~Hz}, 3 \mathrm{H}), 2.29(\mathrm{br} \mathrm{s}, 1 \mathrm{H}), 3.67(\mathrm{~m}, 1 \mathrm{H}), 3.83(\mathrm{~m}, 1 \mathrm{H}), 3.93(\mathrm{~m}$, $1 \mathrm{H}), 7.46-7.61(\mathrm{~m}, 3 \mathrm{H}), 7.95-7.98(\mathrm{~m}, 2 \mathrm{H}) ;{ }^{13} \mathrm{C} \mathrm{NMR}\left(\mathrm{CDCl}_{3}\right) \delta=14.5,42.9,64.5,128.4$, 128.7, 133.3, 136.1, 204.4. In this reaction, Sc leaching (41\%) was observed by XRF analysis. 


\section{References}

(1) Okamoto, K.; Akiyama, R.; Yoshida, H.; Yoshida, T.; Kobayashi, S. J. Am. Chem. Soc. 2005, 127, 2125.

(2) Akiyama, R.; Kobayashi, S. J. Am. Chem. Soc. 2003, 125, 3412.

(3) Fujisawa, H.; Nakagawa, T.; Mukaiyama, T. Adv. Synth. Catal. 2004, 346, 1241.

(4) Le Roux, C.; Gaspard-Iloughmane, H.; Dubac, J. Bull. Soc. Chim. Fr. 1993, 130, 832.

(5) Komoto, I.; Kobayashi, S. J. Org. Chem. 2004, 69, 680.

(6) Iimura, S.; Manabe, K.; Kobayashi, S. Tetrahedron 2004, 60, 7673.

(7) Akiyama, T.; Takaya, J.; Kagoshima, H. Adv. Synth. Catal. 2002, 344, 338.

(8) Okitsu, O.; Oyamada, H.; Furuta, T.; Kobayashi, S. Heterocycles 2000, 52, 1143.

(9) Manabe, K.; Oyamada, H.; Sugita, K.; Kobayashi, S. J. Org. Chem. 1999, 64, 8054.

(10) Nakajima, M.; Yamamoto, S.; Yamaguchi, Y.; Nakamura, S.; Hashimoto, S. Tetrahedron 2003, 59, 7307.

(11) Ozaki, Y.; Kubo, A.; Okamura, K.; Kim, S.-W. Chem. Pharm. Bull. 1995, 43, 734.

(12) This monomer was prepared from 1,12-dodeca-dicarboxylic acid monoethylester ${ }^{13}$ via three steps (Friedel-Crafts acylation with benzene, Wittig reaction with methyltriphenylphosphonium bromide, and LAH reduction of the terminal ester (total yield; $25 \%$ ). White powder; mp $34-35{ }^{\circ} \mathrm{C} ;{ }^{1} \mathrm{H}$ NMR $\left(\mathrm{CDCl}_{3}\right) \delta=$ 1.18-1.60 (m, 23H), $2.49(\mathrm{~m}, 2 \mathrm{H}), 3.63(\mathrm{t}, J=6.4 \mathrm{~Hz}, 2 \mathrm{H}), 5.04(\mathrm{~s}, 1 \mathrm{H}), 5.25(\mathrm{~s}, 1 \mathrm{H}), 7.22-7.43(\mathrm{~m}, 5 \mathrm{H}) ;{ }^{13} \mathrm{C}$ $\operatorname{NMR}\left(\mathrm{CDCl}_{3}\right) \delta=25.7,28.2,29.3,29.4,29.6$ (including 6C), 32.8, 35.3, 63.1, 111.9, 126.1, 127.2, 128.2, 141.5, 148.8; IR (neat) 3320, 2921, $2848 \mathrm{~cm}^{-1}$; HRMS (FAB) Exact mass calcd for $\mathrm{C}_{21} \mathrm{H}_{35} \mathrm{O}[\mathrm{M}+\mathrm{H}]^{+}$, 303.2688, Found 303.2703.

(13) Siatra-Papastaikoudi, T.; Papadaki-Valiraki, A.; Tsantili-Kakoulidou, A.; Tzouvelekis, L.; Mentis, A. Chem. Pharm. Bull. 1994, 42, 392.

(14) Manabe, K.; Ishikawa, S.; Hamada, T.; Kobayashi, S. Tetrahedron 2003, 59, 10439. 